\title{
Superabsorbent and fully bio-based protein foams with a natural crosslinker and cellulose nanofibers
}

\author{
Antonio J. Capezza ${ }^{\mathrm{a}, \mathrm{b}}$, Qiong Wu $\mathrm{W}^{\mathrm{a}}$, William R. Newson ${ }^{\mathrm{b}}$, Richard T. Olsson ${ }^{\mathrm{a}}$, Eliane \\ Espuche $^{\mathrm{c}}$, Eva Johansson ${ }^{\mathrm{b}}$, Mikael S. Hedenqvist $\mathrm{t}^{\mathrm{a}}$
}

${ }^{a}$ KTH Royal Institute of Technology, Teknikringen 56, School of Engineering Sciences in Chemistry, Biotechnology and Health, Fibre and Polymer Technology, SE-100 44

Stockholm, Sweden

${ }^{b} S L U$ Swedish University of Agricultural Sciences, Sundsvägen 10 BOX 101, Department of Plant Breeding, SE-230 53, Alnarp, Sweden.

${ }^{c}$ Univ Lyon, Université Lyon1, UMR CNRS 5223, Ingénierie des Matériaux Polymères, 15, Bd. André Latarjet, Bâtiment Polytech, 69622 Villeurbanne Cedex, France

Number of pages: 4

Number of figures: 3 


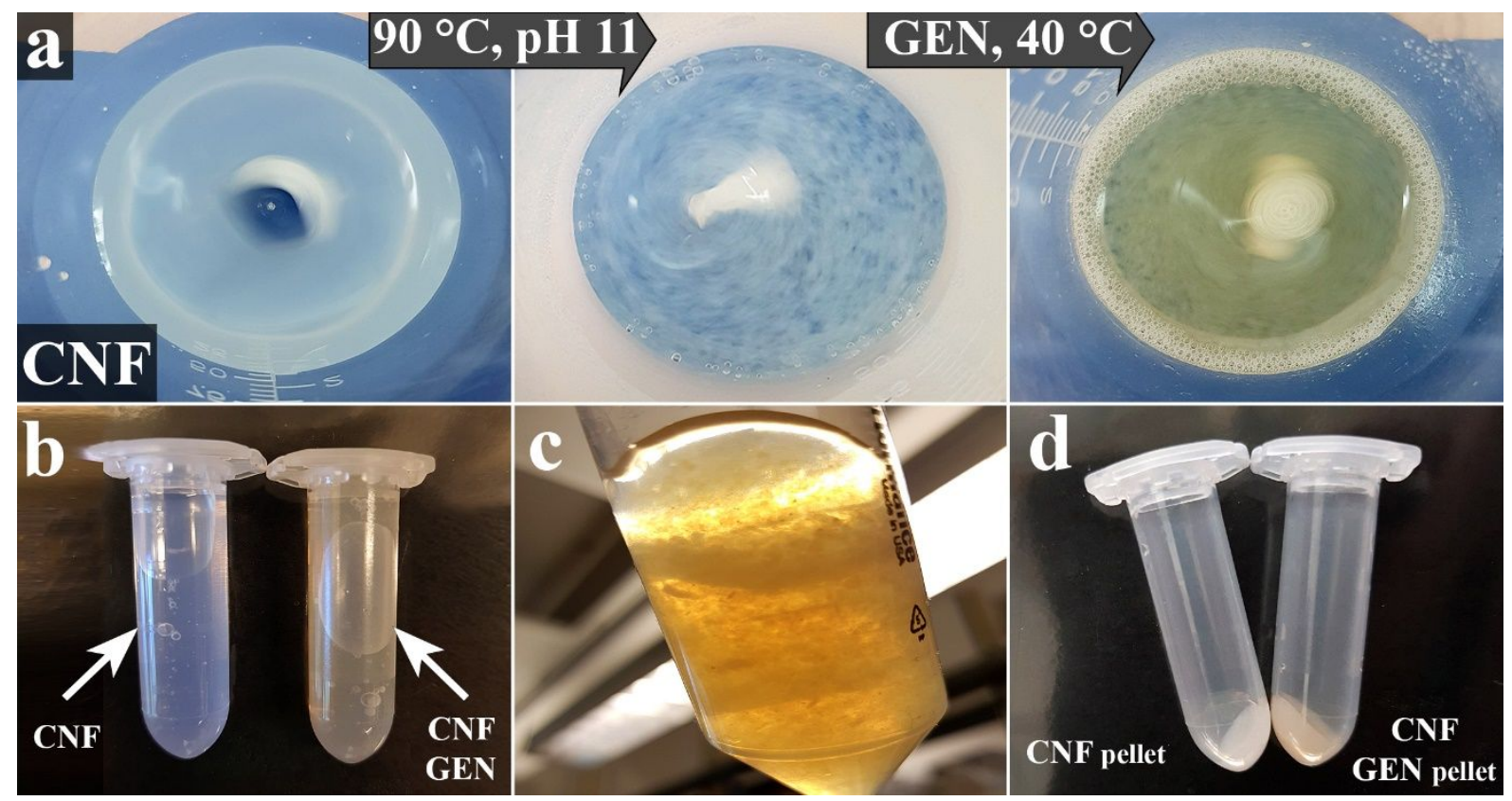

Figure S1: CNF suspensions treated with genipin following the standard protocol but without wheat gluten (a). $50 \mathrm{mg}$ of CNF were dispersed in MQw, thereafter the $\mathrm{pH}$ was adjusted to 11 and the temperature raised to $90{ }^{\circ} \mathrm{C}$, followed by an addition of $50 \mathrm{mg}$ GEN at $40{ }^{\circ} \mathrm{C}$ and stirring during $2 \mathrm{~h}$. The original CNF suspension and the final 
CNF/GEN suspension, the phase separation of the CNF/GEN suspension and the pellet of CNF and CNF/GEN are shown in b, $c$ and $d$, respectively. 


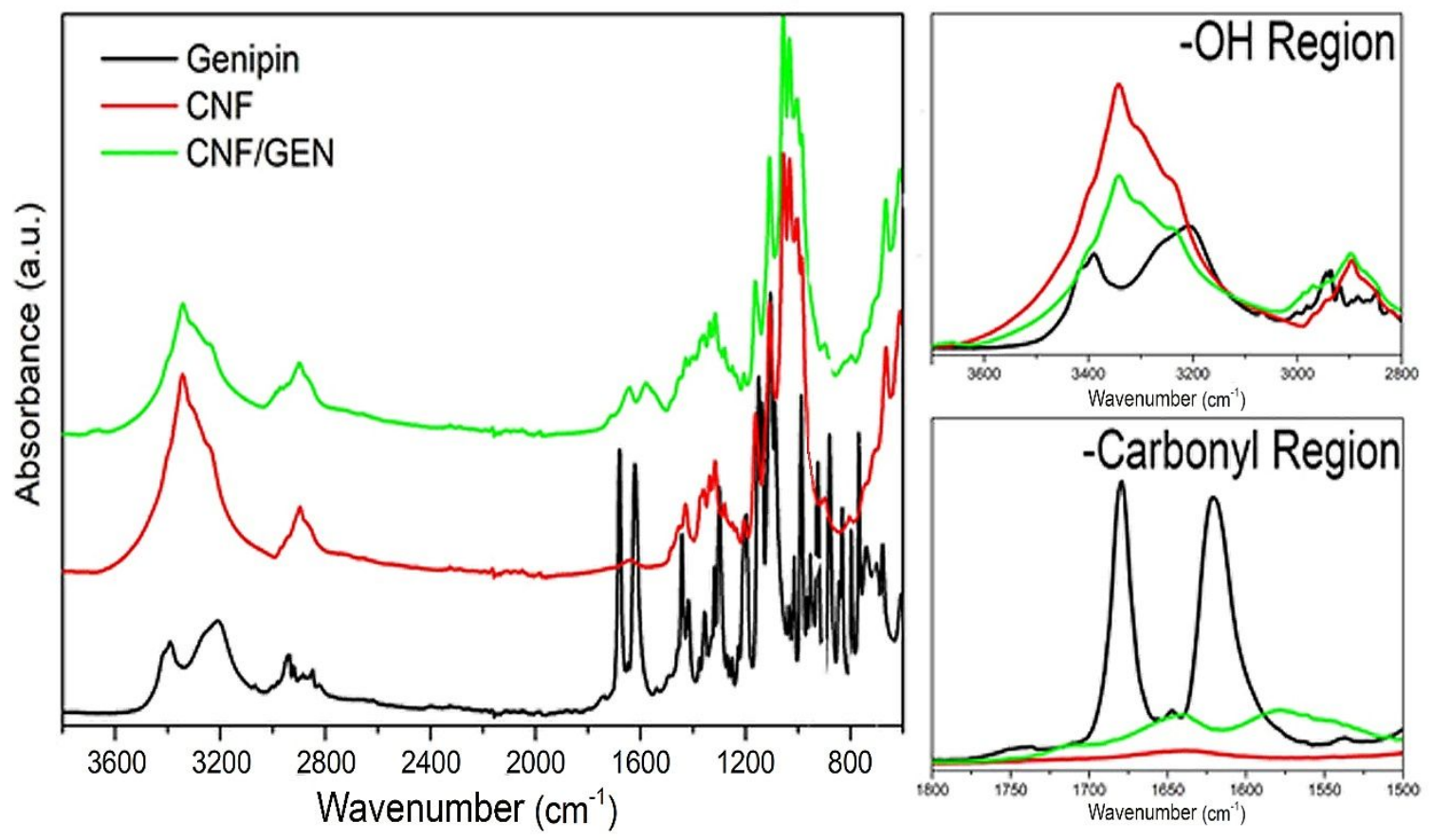

Figure S2: FT IR of the neat CNF, GEN and CNF reacted with GEN (CNF/GEN). 

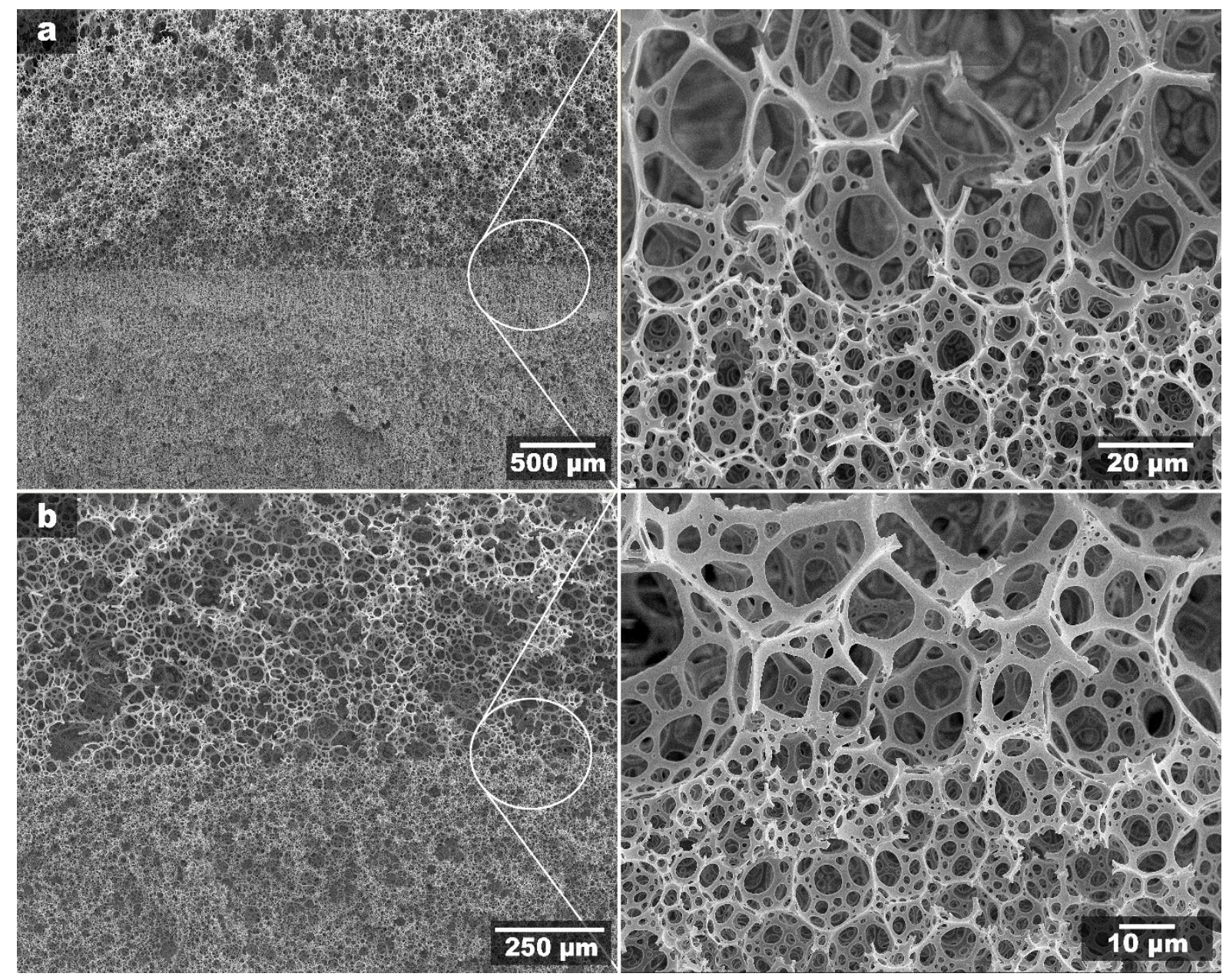

Figure S3: SEM micrographs of (a) commercial foam taken from a sanitary pad and

(b) the freeze-dried structure of the foam after $24 \mathrm{~h}$ immersion in water.

Video S1: swelling behaviour of the WG/CNF/GEN foam after 10 min swelling in sheep blood compared to a commercial foam taken from a sanitary pad.

Video S2: elastic recovery of the W/CNF/GEN foams after $24 \mathrm{~h}$ swelling. 
Conflicts of interest.

No conflicts of interest to declare. 